\title{
Biomarkers in sepsis
}

\author{
Mervyn Singer
}

\section{Purpose of review}

This review discusses the current developments in biomarkers for sepsis.

\section{Recent findings}

With quantum leaps in technology, an array of biomarkers will become available within the next decade as point-of-care tools that will likely revolutionize the management of sepsis. These markers will facilitate early and accurate diagnosis, faster recognition of impending organ dysfunction, optimal selection and titration of appropriate therapies, and more reliable prognostication of risk and outcome. These diagnostics will also enable an improved characterization of the biological phenotype underlying sepsis and thus a better appreciation of the condition.

\section{Summary}

The potential for novel biomarkers in sepsis will need to be properly realized with considerable funding, academic-industry collaborations, appropriate investigations and validation in heterogenous populations, but these developments do hold the capacity to transform patient care and outcomes.

\section{Keywords}

biomarker, diagnosis, prognostication, sepsis, therapy selection

\section{INTRODUCTION}

The recent inability of activated protein $C$ to show an outcome benefit in a randomized controlled multicentre trial $\left[1^{\prime \prime}\right]$ and the subsequent withdrawal of the product from commercial use add to the growing stockpile of failed therapeutics for sepsis. Billions of dollars have been spent on such drugs that have predominantly focussed on one form or another of modulation of the systemic inflammatory/immune response underlying sepsis. The likely utility offered by these agents to some individual patients has been diluted down in these large heterogenous trial populations, either by a large number of patients in whom no benefit was achieved or, potentially, by a near-equivalent number of patients who were harmed. In general, these failed drugs have been consigned to a rear shelf, but on occasion new roles have been found. The most stunning example to date is anti-tumour necrosis factor (TNF) antibody therapy, a dismal flop in sepsis but a gigantic success in rheumatoid arthritis and psoriasis. Indeed, anti-TNF antibodies constitute the top three pharmaceutical best sellers of 2012 with predicted sales in excess of $\$ 26$ billion [2]: impressive progress after an inauspicious start.

The realization is slowly dawning that reliance on a clinical phenotype of severe sepsis or septic shock, a strategy that has essentially remained unchanged for over two decades, is fundamentally flawed [3]. Greater insights into the disease pathophysiology have highlighted the lack of agreement between clinical presentation and immune phenotype. Indeed, many critically ill patients exhibit an overall anti-inflammatory mediator balance even on admission to an intensive care unit (ICU) [4]. The likelihood of an advantageous response from further immunosuppression is thus improbable. Arguably, such patients could gain from judicious immune stimulation. This has been demonstrated in small randomized trials using either granulocytemacrophage colony stimulating factor [5] or interferon-gamma [6], in which a concurrent improvement in immune cell functionality mirrored the clinical improvement in previously static patients.

This review article highlights four areas of recent high-level activity, namely early identification of

Professor of Intensive Care Medicine, University College London, London, UK

Correspondence to Mervyn Singer, Professor of Intensive Care Medicine, Bloomsbury Institute of Intensive Care Medicine, University College London, Cruciform Building, Gower Street, London WC1E 6BT, UK. Tel: +44 207679 6714; e-mail: m.singer@ucl.ac.uk

Curr Opin Pulm Med 2013, 19:305-309 DOI:10.1097/MCP.0b013e32835f1b49

This is an open access article distributed under the Creative Commons Attribution License 4.0, which permits unrestricted use, distribution, and reproduction in any medium, provided the original work is properly cited. 


\section{KEY POINTS}

- Current management of sepsis is complicated, and patient outcomes are compromised, by the difficulties in diagnosis, delayed recognition of organ dysfunction and a poorly characterized biological phenotype.

- The development of new and effective therapies for sepsis has been severely hampered by an inability to select individual patients who will most likely benefit from such treatments, and to precisely titrate dosing and duration.

- Within the next decade, new technologies promise rapid point-of-care testing that should considerably augment diagnostic, therapeutic and prognostic capabilities.

- Considerable research is required to properly validate these tools and confirm their utility.

sepsis, early identification of organ dysfunction, biomarkers to indicate the likely efficacy of a strategy and prognosticators of outcome.

\section{EARLY IDENTIFICATION OF SEPSIS}

At present, sepsis is difficult to diagnose with high sensitivity and specificity. The protean manifestations of the condition and the clinical and biochemical similarities to other, noninfective, systemic inflammatory states (e.g. trauma and blood transfusion reaction) present multiple challenges to optimal patient management. Accurately identifying sepsis at an early stage has clearly important ramifications. Not only can appropriate treatments be administered promptly, be it antibiotics or radiological/surgical drainage, but also unnecessary interventions can be avoided. Thus, antibiotic burden can be reduced with concomitant reductions in bacterial resistance, fungal overgrowth, Clostridium difficile infection and the multiple other complications of antibiotics such as renal or hepatic dysfunction. Linking early sepsis recognition with early pathogen detection (and their antibiotic resistance patterns) enables directed and effective therapy to be given sooner, thus maximizing the chances of success.

Many of the traditional markers of infection are notable for their inaccuracy. Neutrophil count and body temperature may be high, normal or low, whereas C-reactive protein levels, reflecting an acute phase hepatic response, may be resolutely normal in the face of overwhelming infection. A high body temperature may also be a reaction to trauma, surgery, various drugs and blood product transfusions. For example, Circiumaru et al. [7] reported that fever (defined as temperature $\geq 38.4^{\circ} \mathrm{C}$ ) affected
70 of 100 consecutive ICU admissions, but only 16 were attributed alone or in part to infection.

Newer biomarkers have their strong advocates, yet systematic reviews are less convincing. Procalcitonin, a prohormone of calcitonin, is one example that is heavily promoted; however, Tang et al. [8] reported a low diagnostic performance for identifying sepsis, with a mean sensitivity and specificity of 71\% [95\% confidence interval (CI) 67-76\%] and an area under the receiver operator characteristic (ROC) curve of 0.78 (95\% CI 0.73-0.83). Ideally, a biomarker should offer values of at 0.90 , if not at 0.95 .

Likewise, a meta-analysis on the diagnostic utility of soluble triggering receptor expressed on myeloid cells-1 (sTREM-1) was recently performed on 11 studies comprising 1795 patients [9]. Pooled sensitivity and specificity were 79\% (95\% CI 65$89 \%$ ) and $80 \%$ (95\% CI 69-88\%), respectively, with an area under the ROC curve of 0.87 (95\% CI $0.84-$ 0.89). The authors of the review concluded that plasma sTREM-1 was inadequate as a single biomarker for diagnosing sepsis.

The challenge with a single inflammatory biomarker is to clearly differentiate an infectious from a noninfectious systemic inflammatory insult. Thus, an immediate postoperative rise in either marker may simply reflect the trauma of surgery, however a maintained level or secondary rise suggests infection in the absence of other causative factors. Groups have proposed combination biomarkers as being more useful. For example, Kofoed et al. [10] analysed 151 patients of whom 96 had bacterial infections. The areas under the curve (AUCs) for the detection of sepsis ranged from 0.50 to 0.81 for, in ascending order, soluble urokinase-type plasminogen activator (suPAR), STREM-1, macrophage migration inhibitory factor (MIF), procalcitonin, neutrophil count and C-reactive protein. Using the six markers as a composite test, the AUC was 0.88 (95\% CI 0.81-0.92), a value significantly greater than that of any single biomarker. Similarly, Gibot et al. [11"'] recently created a 'bioscore' from the serum concentrations of procalcitonin and sTREM-1, and expression of CD64 on neutrophils measured by flow cytometry. This score was superior (AUC 0.97 [95\% CI 0.95-0.99]) to any of the markers used alone (AUC ranging from 0.730.95); in a validation cohort of 79 patients, $90.9 \%$ were correctly classified by this bioscore.

Perhaps the next generation of sepsis diagnosis markers will rely more on alternative approaches. For example, preliminary transcriptomic analyses have identified increased or decreased expression of genes encoding for proteins in a variety of often disparate pathways that appear to be a characteristic 
of sepsis. In 92 ICU patients at risk of developing sepsis, Lukaszewski et al. [12] took daily white cell samples for the measurement of changes in the expression of interleukin (IL)-1 $\beta$, IL-6, IL-8, IL-10, TNF- $\alpha$, FasL and CCL2 mRNA by real-time reverse transcriptase PCR. Neural networks using these cytokine and chemokine data correctly predicted the development of sepsis in $83 \%$ of cases at 1-4 days before the clinical diagnosis was made; this was achieved with high sensitivity and selectivity (91.4 and $80.2 \%$, respectively). In a separate study, Sutherland et al. [13"] performed leucocyte multigene array and multiplex-tandem PCR studies to assess the transcriptional profiles in healthy volunteers and in ICU patients with sepsis or postoperatively. Using a panel of 42 genes, sepsis could be predicted with an area under the ROC curve ranging from 86 to $92 \%$.

Another interesting development is the ability to differentiate between different septic transcriptomes. Maslove et al. [14] recently analysed neutrophils from patients meeting traditional diagnostic criteria for sepsis to identify two distinct sepsis subtypes based on the expression patterns in 365 genes. This separation was independent of the clinical factors including age, illness severity, mortality and the requirement for organ support. In the first subtype, there was a significant increase in the expression of genes involved in inflammatory and Toll-like receptor mediated signalling pathways, and this was associated with a higher prevalence of severe sepsis. Of interest, expression differed significantly between the subtypes for genes involved in pathways relevant to the actions of drugs used with variable effect in sepsis, including activated protein $\mathrm{C}$, vasopressin, hydrocortisone and norepinephrine.

Such analyses may appear at first sight to be far removed from a rapid diagnostic. However, impressive technological leaps mean that automated, small footprint, point-of-care devices such as multiplex PCR capable of analysing a panel of mRNA transcripts within hours will be a bedside reality within a few years.

\section{EARLY IDENTIFICATION OF ORGAN DYSFUNCTION}

A systemic inflammatory response to infection (sepsis) can lead on to multiple organ dysfunction (severe sepsis). At present, the tools available for clinicians to diagnose organ dysfunction are limited in their capacity to detect early change. Renal dysfunction is currently diagnosed by a fall in urine output and a rise in serum urea and creatinine. However, oliguria may be an appropriate physiological response to hypovolaemia whereas, in patients with previously normal kidneys, creatinine does not rise above normal values until the glomerular filtration rate has fallen by approximately $75 \%$. A vast array of blood and urine biomarkers, of which more than 30 are currently described, can detect decreases in renal function (e.g. cystatin C) or direct tubular stress or injury [e.g. neutrophil gelatinase-associated lipocalin (NGAL)] at a much earlier stage [15]. In addition, biomarkers can be used to predict renal recovery [16"]. At present, it is unclear which biomarkers best prognosticate for significant renal dysfunction and injury, and which can be successfully used to direct therapy to prevent (or at least ameliorate) acute kidney injury.

The same applies to the liver where traditional markers of dysfunction such as hyperbilirubinaemia and coagulopathy occur relatively late, peaking at 3-5 days after the diagnosis of sepsis [17]. However, we have recently shown impaired biotransformation and hepatobiliary transport in a rat model as early as $6 \mathrm{~h}$ after faecal peritoneal contamination [18]. This early dysfunction is supported by a significant rise in plasma bile acids in 48 patients on the day severe sepsis was diagnosed; unlike bilirubin (AUC 0.59), bile acid levels predicted 28-day mortality with high sensitivity and specificity (AUC up to 0.87) [18].

\section{BIOMARKERS TO INDICATE THE LIKELY EFFICACY OF A STRATEGY OR ITS TITRATION}

With the repeated therapeutic failure of immunomodulatory agents for sepsis, there is a growing recognition of the need for biomarkers that can indicate both the likely utility of a drug and how to optimally titrate the dosage [19]. An excellent example is the use of corticosteroids: the use of these agents has vacillated in and out of favour for decades. A revival in the use of corticosteroids was stimulated by the finding in a French multicentre study that benefit was restricted to the subset of patients not responding with an appropriate rise in cortisol to an ACTH stimulation test [20]. However, this could not be reproduced in the larger CORTICUS study [21], whilst the wide inter-laboratory and inter-kit variability of ACTH stimulation testing undermined the validity of this test [22]. An interesting laboratory study by Osuchowski et al. [23] found that IL-6 levels taken at $6 \mathrm{~h}$ after a septic insult in mice could differentiate between responders and nonresponders to dexamethasone, with significant outcome benefit in the high IL-6 group, and a trend towards harm in 
those with low IL-6 levels. Similar findings were recently reported in dogs [24].

Perhaps, a good biomarker for activated protein C may have targeted those patients who could have benefitted from the therapy. A retrospective analysis of plasma levels of protein C activity suggested worse outcomes in those septic patients with low levels on study enrolment and in particular those whose levels failed to normalize over time [25]. A better response was achieved in those patients who had received activated protein $\mathrm{C}$ therapy. This provided a biologic rationale for titrating therapy; however, a phase II trial in which therapy was titrated according to the levels failed to support the utility of protein $\mathrm{C}$ activity as a biomarker [26].

Although the sensitivity/specificity of procalcitonin precludes its use as a stand-alone sepsis diagnostic [8], several studies have used it to allow earlier cessation of antibiotic therapy [27], though reductions in antibiotic consumption have not always been achieved [28]. Arguably, a negative test provides a comfort blanket to clinicians to provide the necessary confidence to cease antibiotics, though the infection may have been eradicated well beforehand. Of note, a recent study using daily procalcitonin measurements to trigger a drug-escalation algorithm and intensified diagnostics resulted in significantly greater antibiotic use, but no improvement in survival, more organ failure and a greater length of stay in the ICU [29].

\section{PROGNOSTIC BIOMARKERS}

Many biomarkers have been shown to be prognostic for the eventual outcome in ICU patients. Few studies have been multicentre or large scale but do imply the dye is cast at an early stage in the patient's presentation to hospital. A multicentre study of 1886 patients hospitalized with community-acquired pneumonia revealed a strong association between plasma cytokine levels and 90-day mortality, with the worst outcomes seen in the subset with elevated levels of both pro-inflammatory and anti-inflammatory cytokines (IL-6 and IL-10, respectively) [30]. Another multicentre study performed in 10 emergency departments found a biomarker panel of NGAL, protein C and IL-1 receptor antagonist predicted severe sepsis, septic shock and death with AUCs for accuracy of $0.80,0.77$ and 0.79 , respectively [31].

Other studies have shown prognostic capability from biomarkers of physiological or biochemical perturbation: for example, heart rate [32], lactate [33,34] and autonomic dysfunction [35]; immunological, inflammatory, coagulation and endothelial activation markers [36-41]; markers of bioenergetic- metabolic abnormality [33,34,42-44]; hormone levels $[37,45,46]$; markers of organ function/damage, for example, echocardiographic markers [47], troponins [48], B-type natriuretic peptides [49] and markers of brain injury [50]; and a range of standalone biomarkers including plasma DNA, nucleated red blood cells and faecal $\mathrm{pH}$ [51-53].

Importantly, none have shown the ability to completely differentiate between survivors and nonsurvivors. These markers represent a wide range of perturbed body systems and this underlies the complex, multisystem pathophysiology of the condition.

\section{CONCLUSION}

New biomarkers will revolutionize the manner in which sepsis is managed, in terms of early recognition, targeting and titration of therapy, and prognostication. Point-of-care technologies will facilitate both measurement and patient management. It is also likely to benefit trial design and enable putative therapies to be better evaluated. For example, survival studies can be focussed upon those people predicted to be at high risk of dying, whereas a specific immunomodulatory agent can be targeted at those with raised or depressed levels, as appropriate. Importantly, these biomarkers need to be validated in large multicentre studies, including heterogenous subsets with respect to age, sex, race, comorbidities, underlying pathogen and source.

\section{Acknowledgements}

None.

\section{Conflicts of interest}

M.S. is on the Scientific Advisory Board of Immunexpress, is the Principal Clinical Investigator of a multicentre biomarker study being undertaken by DSTL (Ministry of Defence), and has given advice on biomarker development in the last 5 years for Abbott, Becton Dickinson, Biomerieux and Fresenius Kabi. The work was undertaken at UCLH/UCL, which received a proportion of funding from the Department of Health's NIHR Biomedical Research Centre's funding scheme.

\section{REFERENCES AND RECOMMENDED \\ READING}

Papers of particular interest, published within the annual period of review, have been highlighted as:

- of special interest

-1 of outstanding interest

Additional references related to this topic can also be found in the Current World Literature section in this issue (p. 329)

1. Ranieri VM, Thompson BT, Barie PS, et al. Drotrecogin alfa (activated) in - adults with septic shock. N Engl J Med 2012; 366:2055-2064.

This trial resulted in the withdrawal of the only licensed drug for sepsis immunomodulation. 
2. Nature Medicine News. Biologic drugs set to top 2012 sales. Nat Med 2012; 18:636.

3. Angus DC. The search for effective therapy for sepsis: back to the drawing board? JAMA 2011; 306:2614-2615.

4. Venet $F$, Davin $F$, Guignant $C$, et al. Early assessment of leukocyte alterations at diagnosis of septic shock. Shock 2010; 34:358-363.

5. Meisel C, Schefold JC, Pschowski R, et al. Granulocyte-macrophage colonystimulating factor to reverse sepsis-associated immunosuppression: a double-blind, randomized, placebo-controlled multicenter trial. Am J Respir Crit Care Med 2009; 180:640-648.

6. Döcke WD, Randow F, Syrbe U, et al. Monocyte deactivation in septic patients: restoration by IFN-gamma treatment. Nat Med 1997; 3:678-681.

7. Circiumaru B, Baldock G, Cohen J. A prospective study of fever in the intensive care unit. Intensive Care Med 1999; 25:668-673.

8. Tang BM, Eslick GD, Craig JC, et al. Accuracy of procalcitonin for sepsis diagnosis in critically ill patients: systematic review and metaanalysis. Lancet Infect Dis 2007; 7:210-217.

9. Wu Y, Wang F, Fan X, et al. Accuracy of plasma sTREM-1 for sepsis diagnosis in systemic inflammatory patients: a systematic review and meta-analysis. Crit Care 2012; 16:R229.

10. Kofoed K, Andersen O, Kronborg G, et al. Use of plasma C-reactive protein, procalcitonin, neutrophils, macrophage migration inhibitory factor, soluble urokinase-type plasminogen activator receptor, and soluble triggering receptor expressed on myeloid cells-1 in combination to diagnose infections: a prospective study. Crit Care 2007; 11:R38.

11. Gibot $S$, Béné $M C$, Noel $R$, et al. Combination biomarkers to diagnose sepsis

I. in the critically ill patient. Am J Respir Crit Care Med 2012; 186:65-71.

A demonstration that a combination biomarker panel is superior as a diagnostic compared with the individual biomarkers.

12. Lukaszewski RA, Yates AM, Jackson MC, et al. Presymptomatic prediction of sepsis in intensive care unit patients. Clin Vaccine Immunol 2008; 15:10891094.

13. Sutherland A, Thomas M, Brandon RA, et al. Development and validation of a

- novel molecular biomarker diagnostic test for the early detection of sepsis. Crit Care 2011; 15:R149.

A transcriptomic approach to diagnose sepsis and differentiate it from other inflammatory conditions, for example, surgery.

14. Maslove DM, Tang BM, McLean AS. Identification of sepsis subtypes in critically ill adults using gene expression profiling. Crit Care 2012; 16:R183.

15. Trof RJ, Di Maggio F, Leemreis J, Groeneveld ABJ. Biomarkers of acute renal injury and renal failure. Shock 2006; 26:245-253.

16. Srisawat $\mathrm{N}$, Wen $\mathrm{X}$, Lee $\mathrm{M}$, et al. Urinary biomarkers and renal recovery in

- critically ill patients with renal support. Clin J Am Soc Nephrol 2011;6:18151823.

A demonstration that urine biomarkers can predict the recovery of renal function.

17. Moreno R, Vincent JL, Matos R, et al. The use of maximum SOFA score to quantify organ dysfunction/failure in intensive care. Results of a prospective, multicentre study. Intensive Care Med 1999; 25:686-696.

18. Recknagel $P$, Gonnert FA, Westermann $M$, et al. Liver dysfunction and phosphatidylinositol-3-kinase signalling in early sepsis: experimental studies in rodent models of peritonitis. PLoS Med 2012; 9:e1001338.

19. Marshall JC, Reinhart K. International Sepsis Forum. Biomarkers of sepsis. Crit Care Med 2009; 37:2290-2298.

20. Annane D, Sébille V, Charpentier $C$, et al. Effect of treatment with low doses of hydrocortisone and fludrocortisone on mortality in patients with septic shock. JAMA 2002; 288:862-871.

21. Sprung $C L$, Annane $D$, Keh $D$, et al. Hydrocortisone therapy for patients with septic shock. N Engl J Med 2008; 358:111-124.

22. Briegel J, Sprung CL, Annane D, et al. Multicenter comparison of cortisol as measured by different methods in samples of patients with septic shock. Intensive Care Med 2009; 35:2151-2156.

23. Osuchowski MF, Connett J, Welch K, et al. Stratification is the key: inflammatory biomarkers accurately direct immunomodulatory therapy in experimental sepsis. Crit Care Med 2009; 37:1567-1573.

24. Hicks CW, Sweeney DA, Danner RL, et al. Beneficial effects of stress-dose corticosteroid therapy in canines depend on the severity of staphylococcal pneumonia. Intensive Care Med 2012; 38:2063-2071.

25. Shorr AF, Bernard GR, Dhainaut J-F, et al. Protein $C$ concentrations in severe sepsis: an early directional change in plasma levels predicts outcome. Crit Care 2006; 10:R92.

26. Shorr AF, Janes JM, Artigas $A$, et al. Randomized trial evaluating serial protein $C$ levels in severe sepsis patients treated with variable doses of drotrecogin alfa (activated). Crit Care 2010; 14:R229.

27. Agarwal R, Schwartz DN. Procalcitonin to guide duration of antimicrobial therapy in intensive care units: a systematic review. Clin Infect Dis 2011; 53:379-387.

28. Layios N, Lambermont $B$, Canivet J-L, et al. Procalcitonin usefulness for the initiation of antibiotic treatment in intensive care unit patients. Crit Care Med 2012; 40:2304-2309.
29. Jensen JU, Hein L, Lundgren $B$, et al. Procalcitonin-guided interventions against infections to increase early appropriate antibiotics and improve survival in the intensive care unit: a randomized trial. Crit Care Med 2011; 39:2048-2058.

30. Kellum JA, Kong L, Fink MP, et al. Understanding the inflammatory cytokine response in pneumonia and sepsis: results of the Genetic and Inflammatory Markers of Sepsis (GenIMS) Study. Arch Intern Med 2007; 167:16551663.

31. Shapiro NI, Trzeciak S, Hollander JE, et al. A prospective, multicenter derivation of a biomarker panel to assess risk of organ dysfunction, shock, and death in emergency department patients with suspected sepsis. Crit Care Med 2009; 37:96-104.

32. Parker SM, Shelhamer JH, Natanson C, et al. Serial cardiovascular variables in survivors and nonsurvivors of human septic shock: heart rate as an early predictor of prognosis. Crit Care Med 1987; 15:923-929.

33. Wacharasint $\mathrm{P}$, Nakada $\mathrm{T}-\mathrm{A}$, Boyd $\mathrm{JH}$, et al. Normal-range blood lactate concentration in septic shock is prognostic and predictive. Shock 2012; 38:4-10.

34. Mtove G, Nadjm B, Hendriksen IC, et al. Point-of-care measurement of blood lactate in children admitted with febrile illness to an African district hospital. Clin Infect Dis 2011; 53:548-554.

35. Schmidt H, Hoyer $D$, Hennen $R$, et al. Autonomic dysfunction predicts both 1 - and 2-month mortality in middle-aged patients with multiple organ dysfunction syndrome. Crit Care Med 2008; 36:967-970.

36. Brenner $\mathrm{T}$, Hofer $\mathrm{S}$, Rosenhagen $\mathrm{C}$, et al. Macrophage migration inhibitory factor (MIF) and manganese superoxide dismutase (MnSOD) as early predictors for survival in patients with severe sepsis or septic shock. J Surg Res 2010; 164:e163-e171.

37. Arnalich F, López J, Codoceo R, et al. Relationship of plasma leptin to plasma cytokines and human survival in sepsis and septic shock. J Infect Dis 1999; 180:908-911.

38. McClintock $D$, Zhuo $H$, Wickersham $N$, et al. Biomarkers of inflammation, coagulation and fibrinolysis predict mortality in acute lung injury. Crit Care 2008; 12:R41.

39. Martin $K$, Borgel $D$, Lerolle $N$, et al. Decreased ADAMTS-13 (a disintegrin-like and metalloprotease with thrombospondin type 1 repeats) is associated with a poor prognosis in sepsis-induced organ failure. Crit Care Med 2007; 35:2375-2382.

40. Que Y-A, Delodder F, Guessous I, et al. Pancreatic stone protein as an early biomarker predicting mortality in a prospective cohort of patients with sepsis requiring ICU management. Crit Care 2012; 16:R114.

41. Ricciuto DR, Santos dos CC, Hawkes M, et al. Angiopoietin-1 and angiopoietin-2 as clinically informative prognostic biomarkers of morbidity and mortality in severe sepsis. Crit Care Med 2011; 39:702710.

42. Hayes MA, Timmins $A C, Y a u E H$, et al. Oxygen transport patterns in patients with sepsis syndrome or septic shock: influence of treatment and relationship to outcome. Crit Care Med 1997; 25:926-936.

43. Brealey $D$, Brand $M$, Hargreaves I, et al. Association between mitochondrial dysfunction and severity and outcome of septic shock. Lancet 2002; 360:219-223.

44. Carré JE, Orban J-C, Re L, et al. Survival in critical illness is associated with early activation of mitochondrial biogenesis. Am J Respir Crit Care Med 2010; 182:745-751.

45. Annane D, Sébille V, Troché $G$, et al. A 3-level prognostic classification in septic shock based on cortisol levels and cortisol response to corticotropin. JAMA 2000; 283:1038-1045.

46. Wang $F$, Pan $W$, Wang $H$, et al. Relationship between thyroid function and ICU mortality: a prospective observation study. Crit Care 2012; 16:R11.

47. Landesberg G, Gilon D, Meroz $Y$, et al. Diastolic dysfunction and mortality in severe sepsis and septic shock. Eur Heart J 2012; 33:895-903.

48. John J, Woodward DB, Wang Y, et al. Troponin-I as a prognosticator of mortality in severe sepsis patients. J Crit Care 2010; 25:270-275.

49. Wang $\mathrm{F}, \mathrm{Wu} \mathrm{Y}$, Tang $\mathrm{L}$, et al. Brain natriuretic peptide for prediction of mortality in patients with sepsis: a systematic review and meta-analysis. Crit Care 2012; 16:R74.

50. Nguyen DN, Spapen H, Su F, et al. Elevated serum levels of S-100beta protein and neuron-specific enolase are associated with brain injury in patients with severe sepsis and septic shock. Crit Care Med 2006; 34:1967-1974.

51. Rhodes A, Wort SJ, Thomas $\mathrm{H}$, et al. Plasma DNA concentration as a predictor of mortality and sepsis in critically ill patients. Crit Care 2006; 10:R60.

52. Stachon A, Segbers $E$, Holland-Letz $T$, et al. Nucleated red blood cells in the blood of medical intensive care patients indicate increased mortality risk: a prospective cohort study. Crit Care 2007; 11:R62.

53. Osuka A, Shimizu $\mathrm{K}$, Ogura $\mathrm{H}$, et al. Prognostic impact of fecal $\mathrm{pH}$ in critically ill patients. Crit Care 2012; 16:R119. 